\title{
Dependence of light pulse propagation on its temporal width: Transition from group velocity to $c$-propagation
}

\author{
Emilio Ignesti, Federico Tommasi *, Lorenzo Fini, Stefano Cavalieri \\ Dipartimento di Fisica e Astronomia, Università di Firenze, via G. Sansone 1, I-50019 Sesto Fiorentino, Italy
}

\section{A R T I C L E I N F O}

\section{Article history:}

Received 8 January 2016

Received in revised form

8 March 2016

Accepted 12 March 2016

Keywords:

Slow light

Pulse propagation

Pulse shaping

Dispersion

\begin{abstract}
A B S T R A C T
We show how the velocity of an optical pulse propagating through a dispersive medium depends on the pulse duration. A transition from the group velocity for long pulses to the in-vacuum velocity for short pulses is shown both in experimental results and in theoretical predictions. The temporal duration of the experimental pulses are $150 \mathrm{ps}$ and $3.5 \mathrm{~ns}$. A description of the pulse propagation in terms of the time "center of mass" of the energy flow allows an intuitive overview of the results.
\end{abstract}

(c) 2016 Elsevier B.V. All rights reserved.

\section{Introduction}

The speed of light in matter has been object of many studies along several decades. Since the work of Brillouin [1] many studies have reported experimental and theoretical results concerning the propagation of a light pulse through a dispersive medium and the possibility of controlling its dynamics by optical methods [2,3], achieving very low velocities [4,5] ("slow light") and also superluminal propagation speed $[6,7]$, but preserving the causality principle [1].

Theoretically, the propagation speed for a light pulse is usually described by the group velocity that is defined as the speed of the wave modulation envelope. Indicating by $n$ the refractive index of the material system, $\omega$ the frequency and $c$ the speed of light in vacuum, the usual expression of the group velocity is the following:

$v_{g}=\frac{c}{n(\omega)+\omega \frac{d n(\omega)}{d \omega}}$

According to this equation, the group velocity is determined by the dispersion properties of the medium at the carrier frequency $\omega$, but it does not depend on other characteristics such as pulse duration or envelope shape, and this is in evident contrast with many experimental observations. Indeed, in this paper we report the measurement of the propagation speed of two optical pulses

\footnotetext{
* Corresponding author.

E-mail address: federico.tommasi@unifi.it (F. Tommasi).
}

with the same carrier frequency but with different durations, and we show that, when traveling through a gaseous medium in conditions of quasi-resonance with an atomic absorption line, the two pulses travel at significantly different speeds without suffering a strong distortion.

The reason of Eq. (1) failure to describe our results is easily understood. This expression is based on a linear approximation of the dispersion relation that demands, to be valid, a narrow bandwidth of the wave packet of the optical signal. When the temporal width of a transform-limited pulse decreases, its spectrum becomes broader, approaching or even completely overlapping the spectral regions where the dispersion relation exhibits large variations, as in the case of a pulse center frequency near to an absorption or gain line in an atomic medium. In the temporal domain, the pulse duration becomes comparable to the response time of the medium; in this case, due to the large frequency spread, the assumption of narrow bandwidth, upon which Eq. (1) is based, becomes not strictly valid and higher order terms in dispersion relation cause distortion of the modulation envelope [8]. As the temporal width further decreases, it is expected that the spectral region of interest, where the dispersion relation rapidly varies, becomes negligible with respect to the pulse bandwidth. In this case the medium response is too slow and the pulse can travel through the system with almost no modification and with a speed that approaches the limit speed $c$.

The traditional way to solve the discrepancy from Eq. (1) and experimental data is to affirm that, in the presence of strong dispersion, the concept of group velocity "has no longer any appreciable physical significance" [9]. A better approach to this problem is to generalize the group velocity concept by introducing a proper 
definition of the pulse propagation time. As an alternative to the peak or half-peak arrival time, the propagation can be described by the average time of the energy flow $[10,11]$, that can be also defined as the time "center of mass" (CM) of the temporal energy envelope [12]. In the case of a pulse propagating along the $z$-axis in an isotropic medium this definition reads:

$t_{C M}(z)=\frac{\int_{-\infty}^{+\infty} t S(z, t) d t}{\int_{-\infty}^{+\infty} S(z, t) d t}$

where $S(z, t)$ is the modulus of the Poynting vector. This equation allows us to achieve a speed definition that is completely meaningful also in presence of strong distortion and outside the validity range of Eq. (1).

Theoretical studies on the dependence on the temporal duration of the superluminal or subluminal propagation have been reported $[13,14]$. Using the average flow arrival time, extensive theoretical works regarding the pulse propagation and reshaping in dispersive media have been reported [15,16]. Experimental studies for ms long pulses in erbium doped fiber [17,18] and for $\mu$ s long pulses via stimulated Brillouin scattering have been reported [19].

In the following, we report for the first time an experimental investigation on the velocity of optical pulses characterized by a temporal durations that fall in an interval between few ns and hundred ps. Pulses of $3.5 \mathrm{~ns}$ and 150 ps of temporal width propagate through an atomic medium where an absorptive resonance is present and a comparison between the experimental results and the numerical simulation is also obtained by integrating the wave equation, showing that the results can be interpreted by a theoretical expression derived from Eq. (2) for the speed of the CM. The aim is to characterize the dependence of the propagation speed on the width of the pulse, also by varying the detuning of the central wavelength of the pulse from the resonance.

Fig. 1 shows experimental temporal profiles, normalized to the peak value, relative to the propagation of two pulses of different FWHM durations: 3500 ps, blue, and 150 ps, red. The dashed lines and the continuous ones are respectively the profiles at the entrance $(z=0)$ and at the end $(z=L=1 \mathrm{~m})$ of the medium, whose description, as well as the experimental setup and method, is reported in Section 3. The propagation times are measured with reference to the CM (dashed vertical lines). Albeit the same experimental conditions, the pulses show two different propagation

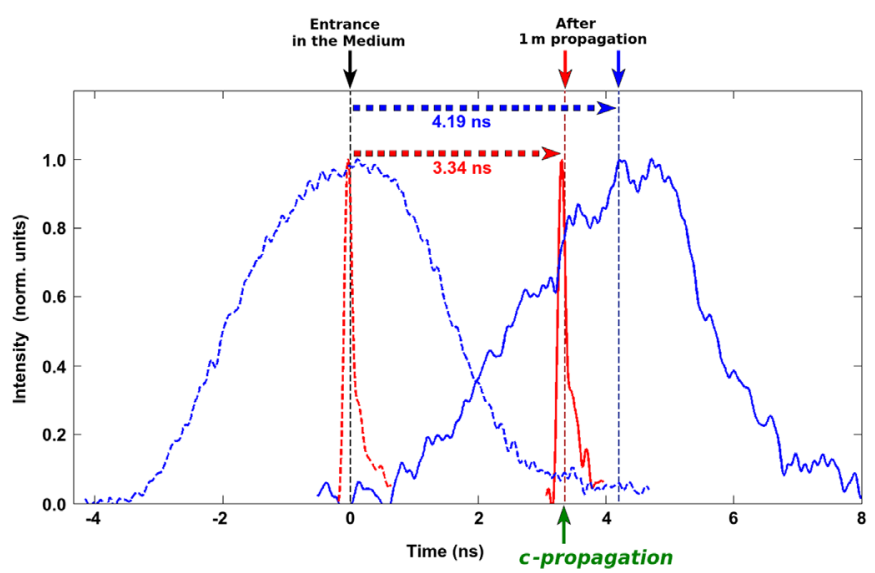

Fig. 1. Experimental profiles of the pulses of width 3500 ps (blue) and 150 ps (red) and central wavelength $\lambda_{p}=819.710 \mathrm{~nm}$. The dashed lines are the pulses at the entrance to cell and the continuous lines are the pulses after the propagation. The dashed vertical lines are the CMs of the pulses. The green arrow below the time axis indicates the propagation at speed $c$ over the same length. (For interpretation of the references to color in this figure caption, the reader is referred to the web version of this paper.)

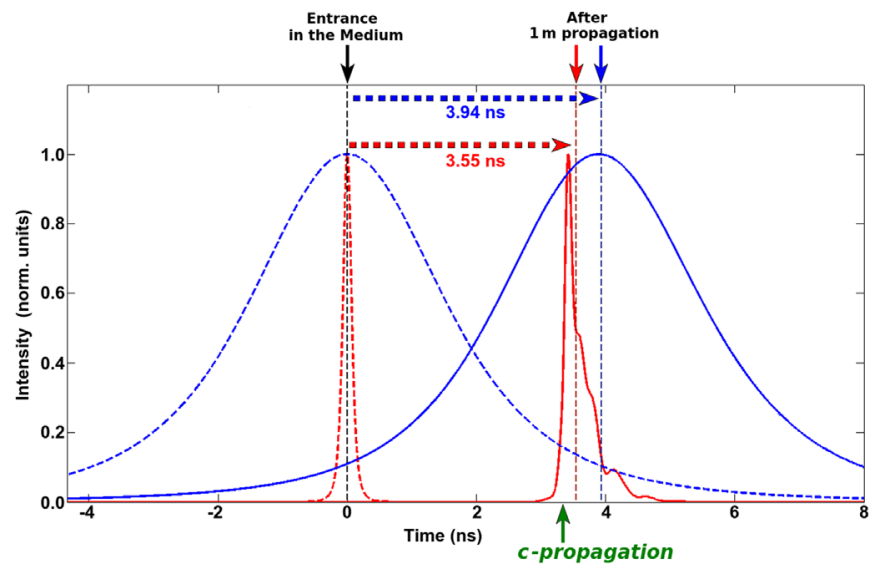

Fig. 2. Numerical simulation for the pulse profiles presented in Fig. 1.

times with the shorter one that appears as nearly unaffected by the medium and exhibits a speed that approaches the speed of light in vacuum. The corresponding numerical simulations are reported in Fig. 2 and a good agreement is present both in shape and in propagation time.

In the following sections the theoretical approaches, the experimental setup and the results are discussed in detail.

\section{Theoretical model}

The propagation of the pulse is given by the solution of the wave equation with a Fourier formalism. For a pulse at the entrance given by:

$E(z=0, t)=\frac{1}{\sqrt{2 \pi}} \int_{-\infty}^{+\infty} \tilde{E}(z=0, \omega) \exp [-i \omega t] d \omega$

the output field at the end of the cell $(z=L)$ is:

$E(z=L, t)=\frac{1}{\sqrt{2 \pi}} \int_{-\infty}^{+\infty} \tilde{E}(z=0, \omega) \exp [i(k(\omega) L-\omega t)] d \omega$

The wave number $k(\omega)$ is a complex quantity that contains informations concerning the dispersion relation and the absorptive properties. With reference to Fig. 3, let us consider an atomic transition between a level $|1\rangle$ and a doublet of levels $|2\rangle$ and $|3\rangle$ and a medium with atomic density $N$. Then, the wave number $k$ has the following expression (low density medium):

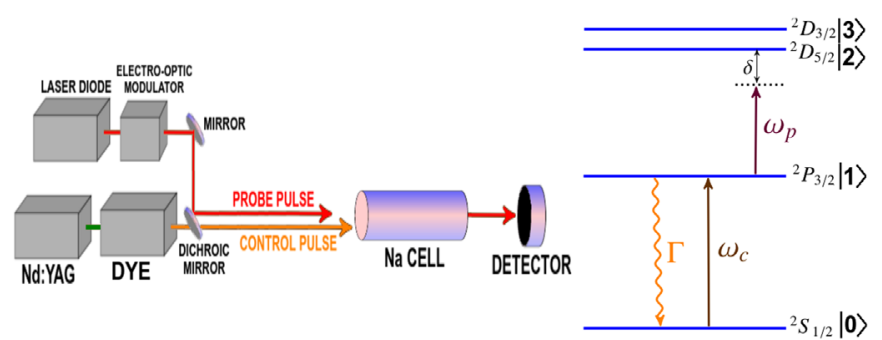

Fig. 3. Schematic diagram of the experimental setup (on the left) and of the involved transitions and sodium atomic levels (on the right). The control pulse is sent into the cell before the probe, whose propagation occurs in an exited medium without the coherent interactions. A dichroic mirror allows the optical paths of the two pulses to coincide before the entrance in the cell. In the atomic interaction scheme on the right, $\omega_{c}$ and $\omega_{p}$ are the central frequencies of the control and the probe pulse respectively, $\Gamma$ the decay rate by spontaneous emission of the level ${ }^{2} P_{3 / 2}$ and $\delta$ is the detuning of $\omega_{p}$ from the resonance with the transition between the level ${ }^{2} P_{3 / 2}$ and the ${ }^{2} D_{5 / 2}$ (the stronger resonance). 
$\kappa(\omega)=\frac{\omega}{c}-\sum_{j=2}^{3} \frac{N \rho_{11} \omega}{4 \hbar \epsilon_{0} c}\left\langle\frac{d_{1 j}^{2}}{i \gamma_{j}+\omega-(v / c+1) \omega_{j 1}}\right\rangle_{v}$

where $\rho_{11}, \gamma_{j}$ and $\omega_{j 1}$ represent, respectively, the lower level population, all kinds of dephasing rates and the $|j\rangle \rightarrow 1$ transition frequencies, whereas the $d_{1 j}$ are the electric dipole moments. The brackets represent the average over the velocity distribution in the $z$ direction and account for the Doppler broadening in the gaseous medium.

In the numerical simulations, the pulse propagation in the temporal domain is obtained by a computation of the Fourier transform based on the Discrete Fourier Transform (DFT) method and a Fast Fourier Transform (FFT) algorithm. Once the temporal position of the $\mathrm{CM}$ of the output pulse with respect to the input one is calculated, the speed can be defined as:

$v_{C M}=\frac{L}{t_{C M}(z=L)-t_{C M}(z=0)}$

An alternative and equivalent theoretical approach consists of directly calculating the CM propagation time by using Eq. (2). As reported in reference [20], this equation can be written in terms of the spectral components of the fields:

$t_{C M}(z)=-i \frac{\int_{-\infty}^{+\infty} \tilde{H}^{*}(z, \omega) \frac{\partial E(z, \omega)}{\partial \omega} d \omega}{\int_{-\infty}^{+\infty} \tilde{H}^{*}(z, \omega) E(z, \omega) d \omega}$

$\tilde{H}^{*}(z, \omega)=\frac{k^{*}(\omega)}{\omega} \tilde{E}^{*}(0, \omega) e^{-i k^{*}(\omega) z}$

Expressing the fields at point $z$ in terms of the fields at $z=0$, we have:

$\tilde{H}^{*}(z, \omega)=\frac{k^{*}(\omega)}{\omega} \tilde{E}^{*}(0, \omega) e^{-i k^{*}(\omega) z}$

and

$t_{C M}(z)=\frac{\operatorname{Im}\left[\int_{0}^{+\infty} \frac{k^{*}}{\omega} e^{-2} \operatorname{Im}(k) z \tilde{E}^{*}(0, \omega) \frac{\partial \tilde{E}(0, \omega)}{\partial \omega} d \omega\right]+\operatorname{Re}\left[\left.\int_{0}^{+\infty} \frac{k^{*}}{\omega} \frac{\partial k}{\partial \omega} \tilde{E}(0, \omega)\right|^{2} e^{-2} \operatorname{Im}(k) z d \omega\right]}{\operatorname{Re}\left[\int_{0}^{+\infty} \frac{k^{*}}{\omega}|\tilde{E}(0, \omega)|^{2} e^{-2 \operatorname{Im}(k) z} d \omega\right]}$

where we have restricted the integration domains to positive frequencies by using the conditions of real fields: $k^{*}(\omega)=-k(-\omega), \quad \tilde{E}^{*}(z, \omega)=\tilde{E}(z,-\omega)$, and $\tilde{H}^{*}(z, \omega)=\tilde{H}(z,-\omega)$. This expression shows that the propagation delay as defined by Eq. (2) can be expressed by the sum of two terms: one is an average of the group delay over the pulse bandwidth and the other takes into account the reshaping due to the absorption or gain properties of the medium [20]. Eq. (10) shows how the propagation characteristics, given by $k(\omega)$ and $\partial k(\omega) / \partial \omega$, are weighted on the overall spectrum of the field. A more intuitive insight is discussed in a simplified form in Section 7.

Then, we have two possible ways to calculate $t_{C M}$ : solving Eq. (4) by the DFT method or using expression (10). Moreover, Eq. (10), rewritten in a simplified form, allows an intuitive solution of the $v_{g}$, as shown in Section 7.

\section{Experimental set-up}

The experimental setup is shown in Fig. 3. The light pulses (probe pulses) are obtained by modulating the continuous-wave beam of a single-mode extended-cavity diode laser by an electrooptic modulator connected to the laser by a monomode optical fiber. Two different electric pulse generators can drive the modulator to produce pulses of two different temporal widths: 3500 and $150 \mathrm{ps}$ (FWHM). The pulses propagate into a $1 \mathrm{~m}$ long cell containing a vapor of sodium atoms, whose density can be varied by controlling the cell temperature. With reference to Fig. 3, the probe pulse frequency is nearly resonant to the sodium transitions from the intermediate level ${ }^{2} P_{3 / 2}$ to the ${ }^{2} D$-doublet (819.7077 and $819.7043 \mathrm{~nm}$ ), a pair of levels separated in frequency by $\sim 1.5 \mathrm{GHz}$. A control pulse, tuned on the transition from the ground state ${ }^{2} S_{1 / 2}$ to the ${ }^{2} P_{3 / 2}$ state, is generated by a frequency tunable dye laser pumped by a frequency-doubled Q-switched Nd:YAG laser at a repetition rate of $10 \mathrm{~Hz}$. The control pulse is used to transfer population to the intermediate level and to induce an interaction between the probe pulse and the atomic system. The pulse is strong enough to saturate the transition (in Eq. (5) $\rho_{11}$ approaches 0.5 ), so that the total population of the involved atoms depends only on the temperature of the cell. A proper delay assures that only incoherent interactions [21-24] between the control and the probe pulse are involved. The advantage of this scheme is that the dispersion properties that affect the probe beam propagation can be switched on or off on demand by the control beam.

After the cell, the temporal profiles are detected by a $12 \mathrm{GHz}$ bandwidth photodiode connected to a 8-GHz-bandwidth digital oscilloscope. A reference measurement session, during which the control pulse is not injected into the cell, allows us to detect the temporal profile of the probe pulse propagating through a nonexcited medium. Under such a condition, due to the very low pressure of the sodium vapor, a propagation in vacuum into the cell can be considered.

\section{Results}

A comparison between experimental and theoretical values of the CM speed for different center wavelengths is shown in Figs. 46 . All detunings are expressed with reference to the stronger resonance $(819.7077 \mathrm{~nm})$. The $v_{C M}$, normalized to $c$ and considering a $1 \mathrm{~m}$ long medium, is shown as a function of the pulse temporal width in the experimental case (filled circles) and for numerical values (empty squares linked with continuous blue lines) calculated by assuming a Lorentzian pulse temporal shape. The dashed red lines represent the limit value at $c$ (above) and the group velocity $v_{g}$ calculated by Eq. (1). To evaluate $v_{g}$ the dispersion curve for the central frequency of the pulse has been calculated from the values of the atomic parameters and the cell temperature.

Different temperatures are used to avoid a severe absorption at small detuning and the effective temperature is the free parameter (around the selected temperature for the cell) in the numerical simulation.

In all numerical cases the CM speed approaches $c$ in the limit of

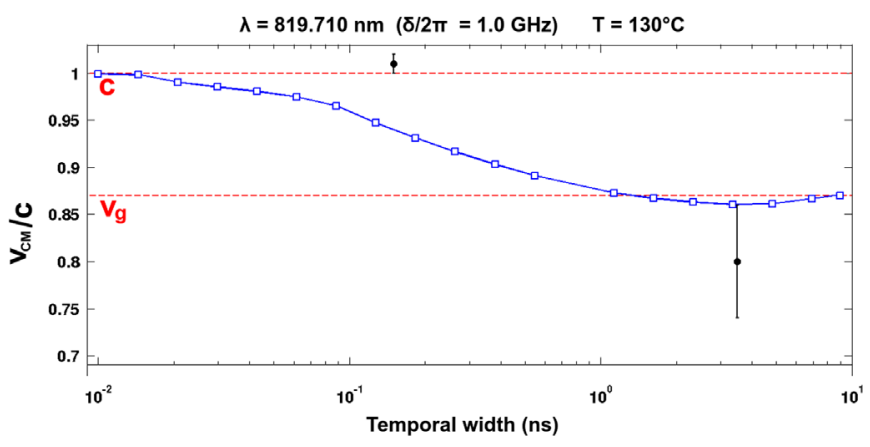

Fig. 4. Normalized "center of mass" speed for $\lambda_{p}=819.710 \mathrm{~nm}$ as a function of the pulse width. The filled circles are experimental data and empty squares are numerical ones. The cell temperature is set to $130^{\circ} \mathrm{C}$. (For interpretation of the references to color in this figure caption, the reader is referred to the web version of this paper.) 


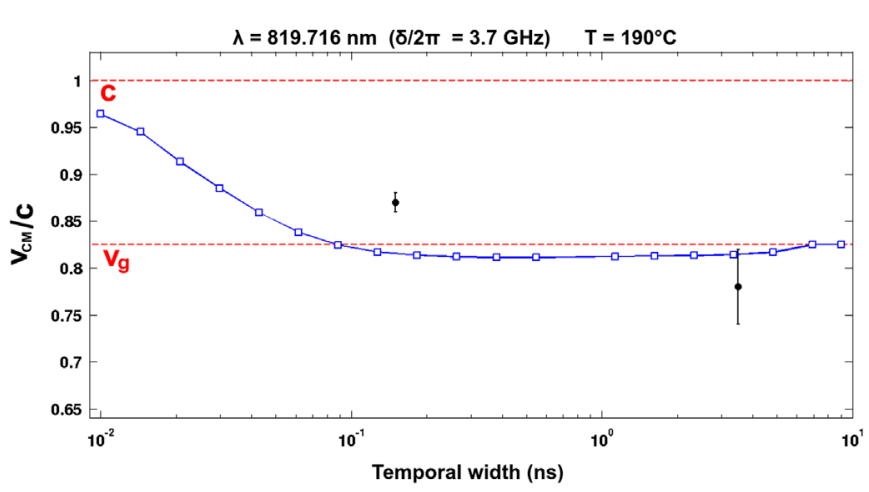

Fig. 5. Normalized "center of mass" speed for $\lambda_{p}=819.716 \mathrm{~nm}$ as a function of the pulse width. The filled circles are experimental data and empty squares are numerical ones. The cell temperature is set to $190^{\circ} \mathrm{C}$. (For interpretation of the references to color in this figure caption, the reader is referred to the web version of this paper.)

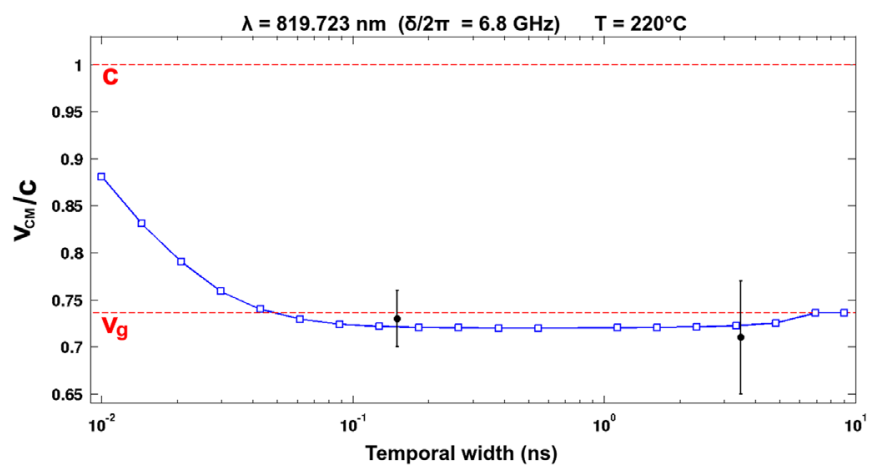

Fig. 6. Normalized "center of mass" speed for $\lambda_{p}=819.723 \mathrm{~nm}$ as a function of the pulse width. The filled circles are experimental data and empty squares are numerical ones. The cell temperature is set to $220^{\circ} \mathrm{C}$. (For interpretation of the references to color in this figure caption, the reader is referred to the web version of this paper.)

very short temporal width and $v_{g}$ in the opposite limit, with a trend that depends on the detuning. Moreover, in the intermediate region $v_{C M}$ can assume superluminal or even sub- $v_{g}$ values. In the detuning range that we have examined no superluminal values are found and the sub- $v_{g}$ speeds at the beginning of the intermediate region may be surprising at a first glance. Although, it can be analytically shown that an expansion of the wave vector $k$ in Taylor series of the frequency leads to an expression of $v_{g}$ where are present the third $\left(k_{3}\right)$ and fifth $\left(k_{5}\right)$ derivative with respect to the frequency (both positive far from resonance):

$v_{C M} \simeq \frac{1}{k_{1}+\frac{k_{3}}{2 d_{t}^{2}}+\frac{k_{5}}{8 d_{t}^{4}}}$

where $d_{t}$ is the temporal width of a Gaussian pulse. Expression (11) is valid only as a correction to a very long pulse.

The experimental data are in large part in agreement with the theoretical predictions. Sources of difference between the theoretical and the experimental behavior are due to terms that have not been included in the numerical simulation. First, a Lorentzian shape has been assumed for the input pulse instead of the real pulse envelope. This has been done to relate the experimental results to a more general physical case. Secondly, an uniform temperature has been assumed instead of the actual temperature in every point of the cell, that is very difficulty to known or to model with enough accuracy. Moreover, the decay of excited atoms during the pulse propagation is not considered in the simulation, as it cannot be included in a frequency-domain calculation in a simple manner. If the pulse duration is of the same

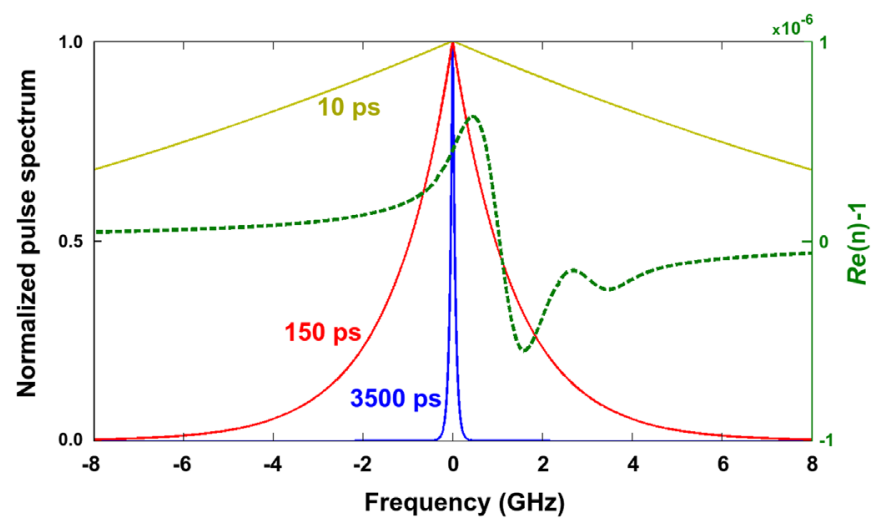

Fig. 7. Numerically calculated spectra of the pulses with two different temporal widths and central wavelength of $819.710 \mathrm{~nm}$. The 0 -frequency is set to the center of the spectra. The real part of the refractive index (dashed green line) is plotted for comparison. (For interpretation of the references to color in this figure caption, the reader is referred to the web version of this paper.)

order of the population decay time, the leading and the trailing edges of the pulse can undergo different propagation velocities and this can lead to a compression of the signal [21,22].

A comparison between the bandwidth of the pulses and the dispersion relation (real part of the refractive index, dashed green line) is shown in Fig. 7, where three different temporal durations for a pulse of Lorentzian shape are reported in the case of central wavelength of $819.710 \mathrm{~nm}$ (case of Fig. 4). Considering a pulse of 3500 ps of temporal duration (blue line), the bandwidth becomes narrow enough to satisfy the requested constrains that underlie Eq. (1); as a consequence, in this case the group velocity becomes a meaningful definition as a propagation speed for this kind of signal. In the red case ( $150 \mathrm{ps}$ of temporal duration), the bandwidth is of the same order of the spectral region where the dispersion varies and can induce a modification on $\mathrm{CM}$ propagation speed. Finally, in the yellow case (10 ps) the extremely large pulse bandwidth leads to a pulse propagation at $c$, as the alteration on the propagation induced by the resonance acts on a negligible part of the pulse.

\section{A simplified picture}

As previously mentioned in Section 2, a more intuitive picture is offered by Eq. (10) in the limit of a low density medium $(n \simeq 1)$. If the absorption is negligible the imaginary part of $k(\omega)$ approaches zero. Then, the CM velocity can be given by:

$\frac{1}{v_{C M}} \simeq \frac{\int_{0}^{+\infty} \frac{\partial k}{\partial \omega}|\tilde{E}(0, \omega)|^{2} d \omega}{\int_{0}^{+\infty}|\tilde{E}(0, \omega)|^{2} d \omega}=\left\langle\frac{1}{v_{g}}\right\rangle$

where the brackets state that $1 / v_{g}$, usually calculated for the central frequency, has to be averaged over the whole spectrum.

In particular, if the pulse spectrum can be approximated by a rectangular shape that differs from zero only within a bandwidth $\delta \omega$ around the central frequency $\omega_{p}$, it can be shown that:

$v_{C M}=\frac{c}{1+\omega_{p} \frac{n\left(\omega_{p}+\delta \omega / 2\right)-n\left(\omega_{p}-\delta \omega / 2\right)}{\delta \omega}}$

With reference to Fig. 7, when the pulse has a large temporal width (3500 ps-case), the bandwidth becomes narrow and expression (13) approaches the usual definition of group velocity given by Eq. (1). On the other hand, when the band becomes broader, as in the case of the 150 ps-pulse, also anomalous 
dispersion zones are probed by frequency components, leading to an averaged effect that increases the propagation speed towards $c$. For a very large bandwidth, the 10 ps-case, the larger contributions come from low dispersive zones far away from the atomic resonance, causing a propagation almost unaffected by the medium response.

All these cases described by the simplified Eq. (13), together with the scenario above described, are qualitatively in agreement with the numerical and experimental results reported in Figs. 4-6. It is worth to note that the approximation is strictly correct only with no frequency selective absorption.

\section{Conclusions}

We have presented a study on the dependence of the "center of mass" speed of an optical pulse on its temporal duration. The experimental results, showing that the shorter pulse is also the faster, are in qualitative agreement with a theoretical investigation. We have shown that the CM speed passes from the group velocity determined by the dispersion curve to the in-vacuum speed $c$ in a range of pulse durations that is determined by the detuning from the absorption resonance.

Moreover, an approximate simplified theoretical picture, here reported, is able to supply an intuitive overview on the modification of the propagation dynamics in the different cases.

\section{Acknowledgments}

The corresponding author acknowledges Cassa di Risparmio di Firenze for supporting his work.

\section{Appendix A. Supplementary data}

Supplementary data associated with this paper can be found in the online version at http://dx.doi.org/10.1016/j.optcom.2016.03. 029.

\section{References}

[1] L. Brillouin, Wave Propagation and Group Velocity, New York Academic, 1960.

[2] R.W. Boyd, D.J. Gauthier, Controlling the velocity of light pulses, Science 326 5956) (2009) 1074-1077, http://dx.doi.org/10.1126/science.1170885.

[3] P. Milonni, Fast. Light, Slow. Light, Left-Handed. Light, Series in Optics and Optoelectronics, CRC Press, 2004
[4] L. Casperson, A. Yariv, Pulse propagation in a high-gain medium, Phys. Rev. Lett. 26 (1971) 293-295, http://dx.doi.org/10.1103/PhysRevLett.26.293.

[5] D. Grischkowsky, Adiabatic following and slow optical pulse propagation in rubidium vapor, Phys. Rev. A 7 (1973) 2096-2102, http://dx.doi.org/10.1103/ PhysRevA.7.2096.

[6] L.J. Wang, A. Kuzmich, D.A. Gain-assisted, superluminal light propagation, Nature 406 (2000) 227-279, http://dx.doi.org/10.1038/35018520.

[7] M.D. Stenner, D.J. Gauthier, M.A. Neifeld, The speed of information in a 'fastlight' optical medium, Nature 425 (2003) 695-698, http://dx.doi.org/10.1038/ nature02016.

[8] R.W. Boyd, D.J. Gauthier, A.L. Gaeta, A.E. Willner, Maximum time delay achievable on propagation through a slow-light medium, Phys. Rev. A 71 (2005) 023801, http://dx.doi.org/10.1103/PhysRevA.71.023801.

[9] M. Born, E. Wolf, Principles of Optics, 6th ed., Cambridge University Press, 1980.

[10] R.L. Smith, The velocities of light, Am. J. Phys. 38 (8) (1970) 978-984, http://dx. doi.org/10.1119/1.1976551.

[11] J. Schwinger, L.L. DeRaad, K.A. Milton, W.-Y. Tsai, Classical Electrodynamics, Perseus Book Group, 1998.

[12] B. Macke, B. Ségard, Propagation of light-pulses at a negative group-velocity, Eur. Phys. J. D-At. Mol. Opt. Plasma Phys. 23 (1) (2003) 125-141, http://dx.doi. org/10.1140/epjd/e2003-00022-0.

[13] L. Nanda, H. Wanare, S.A. Ramakrishna, Why do superluminal pulses become subluminal once they go far enough? Phys. Rev. A 79 (2009) 041806, http://dx. doi.org/10.1103/PhysRevA.79.041806.

[14] A.H. Dorrah, A. Ramakrishnan, M. Mojahedi, Time-frequency dynamics of superluminal pulse transition to the subluminal regime, Phys. Rev. E 91 (2015) 033206, http://dx.doi.org/10.1103/PhysRevE.91.033206.

[15] L. Nanda, A. Basu, S.A. Ramakrishna, Delay times and detector times for optical pulses traversing plasmas and negative refractive media, Phys. Rev. E 74 (2006) 036601, http://dx.doi.org/10.1103/PhysRevE.74.036601.

[16] Z. Zhu, D. Gauthier, Y. Okawachi, J. Sharping, A. Gaeta, R. Boyd, A. Willner Numerical study of all-optical slow-light delays via stimulated Brillouin scattering in an optical fiber, J. Opt. Soc. Am. B 22 (11) (2005) 2378-2384, http: //dx.doi.org/10.1364/JOSAB.22.002378.

[17] F.A.-Y. nez, E. Cabrera-Granado, J.M. Ezquerro, O.G. Calderon, S. Melle, Pulsewidth-dependent subluminal and superluminal propagation in highly doped erbium fibers, J. Opt. Soc. Am. B 28 (5) (2011) 1172-1179, http://dx.doi.org/ 10.1364/JOSAB.28.001172.

[18] A. Schweinsberg, N.N. Lepeshkin, M.S. Bigelow, S. Boyd, R.W. Jarabo, Observation of superluminal and slow light propagation in erbium-doped optical fiber, Europhys. Lett. 73 (2) (2006) 218-224, http://dx.doi.org/10.1209/epl/ i2005-10371-0.

[19] L. Zhang, L. Zhan, M. Qin, Z. Wang, H. Luo, T. Wang, Superluminal propagation through $500 \mathrm{~m}$ optical fiber via stimulated Brillouin scattering, Opt. Lett. 40 (19) (2015) 4404-4407, http://dx.doi.org/10.1364/OL.40.004404.

[20] J. Peatross, S.A. Glasgow, M. Ware, Average energy flow of optical pulses in dispersive media, Phys. Rev. Lett. 84 (2000) 2370-2373, http://dx.doi.org/ 10.1103/PhysRevLett.84.2370.

[21] M.V. Tognetti, E. Sali, S. Cavalieri, R. Buffa, Temporal pulse compression and retardation by incoherent all-optical control, Phys. Rev. A 81 (2010) 023807 , http://dx.doi.org/10.1103/PhysRevA.81.023807.

[22] E. Ignesti, F. Tommasi, R. Buffa, L. Fini, E. Sali, M.V. Tognetti, S. Cavalieri, Incoherent optical control of pulse propagation and compression, Phys. Rev. A 86 (2012) 063818, http://dx.doi.org/10.1103/PhysRevA.86.063818.

[23] E. Ignesti, F. Tommasi, R. Buffa, L. Fini, E. Sali, S. Cavalieri, Optical control of superluminal propagation of nanosecond laser pulses, Phys. Rev. A 87 (2013) 033828, http://dx.doi.org/10.1103/PhysRevA.87.033828.

[24] F. Tommasi, E. Ignesti, L. Fini, S. Cavalieri, Recovering the propagation delay of an optical pulse, Opt. Express 22 (23) (2014) 28566-28571, http://dx.doi.org/ 10.1364/OE.22.028566. 